\title{
Frequent Premature Ventricular Complexes by ECG Finding
}

National Cancer Institute

\section{Source}

National Cancer Institute. Frequent Premature Ventricular Complexes by ECG Finding. NCl Thesaurus. Code C120611.

An electrocardiographic tracing demonstrating frequent premature ventricular contractions that exceed an acceptable, predetermined threshold. Different thresholds may apply to different patient populations. 